\title{
Variability of Cosmic Ray Power Spectra
}

\author{
K. KUDELA ${ }^{1}$, D. VenKATESAN ${ }^{2}$, and R. LANGER ${ }^{1}$ \\ ${ }^{1}$ Institute of Experimental Physics, SAS, Kosice, Slovakia \\ ${ }^{2}$ Department of Physics and Astronomy, University of Calgary, Canada
}

(Received October 4, 1995; Revised March 4, 1996; Accepted April 1, 1996)

\begin{abstract}
Variability of the power spectra of cosmic ray temporal profiles in the frequency interval $1.2 \times$ $10^{-5} \mathrm{~Hz}\left(f_{1}\right)$ to $1.7 \times 10^{-3} \mathrm{~Hz}\left(f_{2}\right)$ in the period of two years shows that the integrals of power spectrum density over the interval $f_{1}$ to $2.8 \times 10^{-4} \mathrm{~Hz}\left(P_{1}\right)$ are significantly correlated at two neutron monitors differing in acceptance cones and cut-off rigidities. Athigh frequency region, $f>2.8 \times 10^{-4} \mathrm{~Hz}$, the integrals $P_{2}$ are correlated much less. $P_{1}$ are weakly correlated with the cosmic ray intensity itself. Cross correlation between the time series $P_{1}$ and the daily averages of geomagnetic indices $(\overline{K p}, \overline{D s t}$ and $A p)$ is asymmetric, with the maximum shifted to positive time lag. This indicates the stronger relation of the daily averaged geomagnetic indices measured for given day with the $P_{1}$ obtained a day before, than with the $P_{1}$ at the same day. The shift is not obtained if cosmic ray intensity, instead of $P_{1}$, is cross-correlated with geomagnetic indices.
\end{abstract}

\section{Introduction}

Besides the clear periodic and quasiperiodic variations of cosmic ray (CR) intensity recorded by neutron monitors (NM), like the 11-year and the diurnal variation, the superposition of small amplitude variations, the fluctuations, lead to the observed broad-band power spectrum of CR. The fluctuations of CR (CR scintillations) were first discussed by Dhanju and Sarabhai (1967) and by Jokipii (1969). The general characteristics of CR fluctuations and their relation to magnetospheric and interplanetary phenomena were described in a series of papers by Owens and Jokipii (cited below). The CR scintillations at neutron monitor (NM) energies are not explained by magnetospheric processes, in difference to lower energy particles, and may be of interplanetary origin Owens and Jokipii (1972). The equation relating the CR scintillations to interplanetary magnetic field (IMF) fluctuations and to CR gradients was obtained in Owens (1974). Thus, the CR scintillations may provide useful information about interplanetary fields. A model for the production of CR scintillations by IMF irregularities developed in Owens and Jokipii (1974) has shown good agreement with the observed CR scintillations $\sim 1 \mathrm{GeV}$ kinetic energy. Further, the crosscorrelation between $\mathrm{CR}$ fluctuations and IMF confirmed earlier theory and provided good explanation of the observed NM fluctuations and their correlation with the IMF in the range $5 \times 10^{-6} \mathrm{~Hz}<f<2 \times 10^{-5}$ Hz Jokipii and Owens (1974).

The comparison of the theory with the observations in the referenced papers was based on the 90 day quiet period in 1969 as measured by Alert and Deep River NM. During the periods of interplanetary disturbances, when IMF is significantly redistributed, the changes of power spectrum density (PSD) of CR fluctuations are expected. Our analysis is devoted to the variability of PSD characteristics of CR and based on 5-minute data from two NMs over relatively long time period of two years, during which both the quiet intervals, as well as the strongest interplanetary disturbances over the cycle 22 (i.e., March 1991 and June 1991, see e.g., Shea et al., 1993) were observed. We analyze the similarities in the PSD in the range $1.2 \times 10^{-5} \mathrm{~Hz}<f<1.7 \times 10^{-3} \mathrm{~Hz}$ at the two $\mathrm{NMs}$ with the aim (i) to find a suitable parameter characterizing the variability of CR fluctuations common for NM energies $\left(P_{1}\right)$, and (ii) to check the relation between the parameter of the $\mathrm{CR}$ fluctuations and the indices characterizing the geomagnetic 
activity. We will show potential usefulness of the $P_{1}$ for including it as one of the parameters into the schemes dealing with the prediction of geomagnetic disturbances.

\section{Characteristics of CR Fluctuations}

5-minute pressure corrected data during the years 1991-1992 are used for the analysis of CR fluctuation characteristics, as measured by two NMs, namely (i) Calgary (CG, $51^{\circ} 05^{\prime} \mathrm{N}, 114^{\circ} 08^{\prime} \mathrm{W}$, vertical cut-off rigidity $R c=1.1 \mathrm{GV}$, count rate $n \sim 2 \times 10^{4}$ per 5 minute) and (ii) Lomnicky Stít (LS, $49^{\circ} 20^{\prime} \mathrm{N}$, $20^{\circ} 22^{\prime} \mathrm{E}, R c=4.0 \mathrm{GV}$, count rate $n \sim 1.5 \times 10^{5}$ per $5 \mathrm{~min}$ ). PSD is characterized for each day at the two stations. Out of total 731 days, 685 days are fully covered by the measurements simultaneously at both NMs. Two examples of PSD at different days at LS are shown in Fig. 1. Data are normalized (the daily mean is removed) and detrended by the linear function which is substantial in assuming the approach of stationary time series. The detrending is essential during the intervals of strong low frequency variations like Forbush decreases. The use of higher order polynomials for detrending leads to not significantly different values of PSD. PSD in Fig. 1 is obtained using the indirect method based on Fourier transform

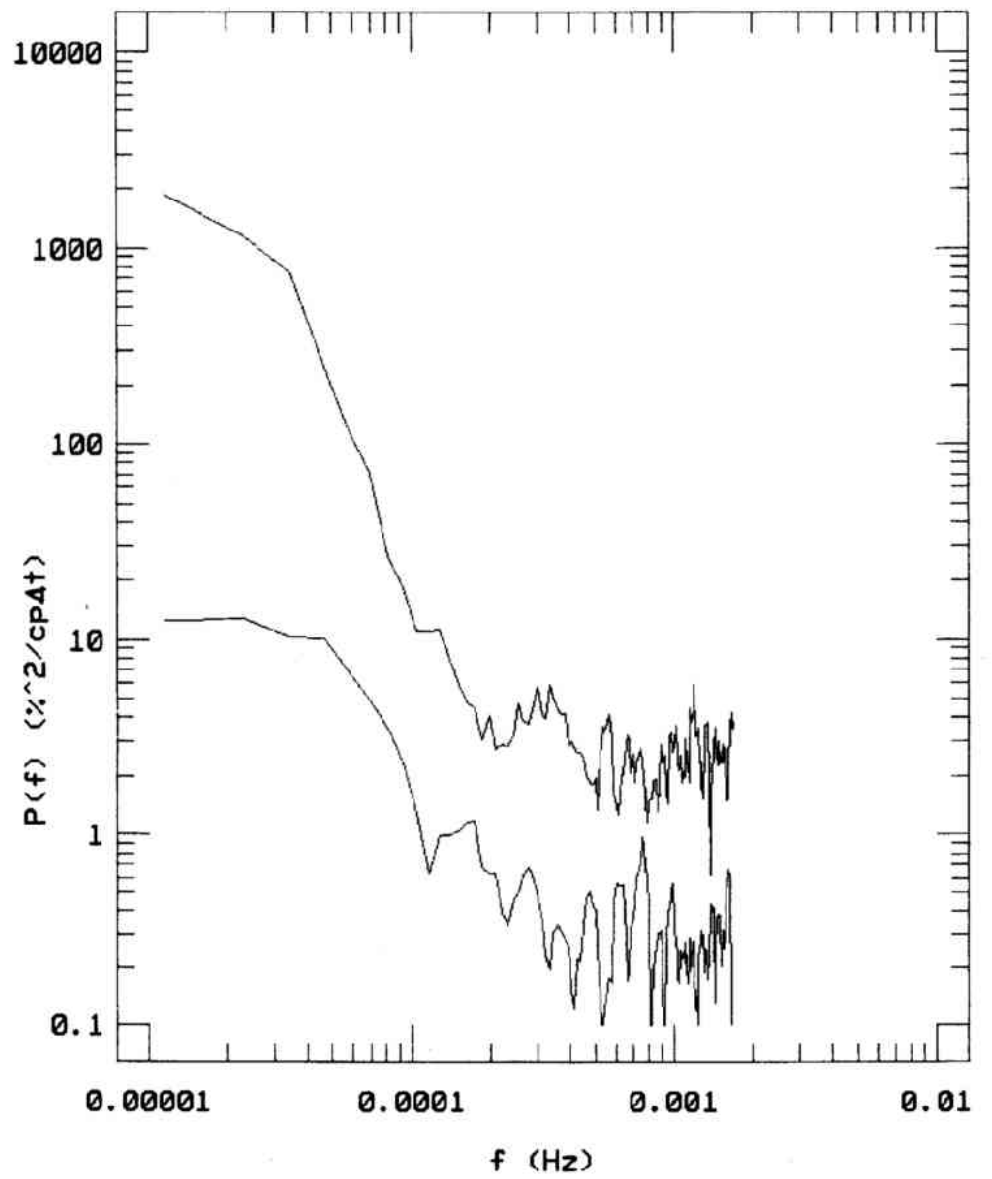

Fig. 1. Power spectrum density (PSD, in units $(\%)^{2} /($ cycles per $\Delta t), \Delta t=10$ minutes of CR intensity at LS for the day $197 / 1991$ (lower curve) and for day $137 / 1991$ (upper curve, multiplied by 10), illustrating the variability of PSD at low frequencies. The level of Poisson noise is 0.075 in these units for LS ( 0.205 for CG). For the computations the series of 5 min resolution linearly detrended data are used. 
of autocorrelation function, or periodogram Box and Jenkins (1970). The difference in the two PSD is apparent especially in the low frequency region, $f<10^{-4} \mathrm{~Hz}$. At the higher frequencies, two effects may become important: (i) the action of IMF on CR is ineffective, and (ii) the Poisson noise affects the computed PSD. To assume the highest frequency, above which the IMF can not cause CR fluctuations at NM energies, we adopted the approach of Bazilevskaya and Struminsky (1993): in the diffusion approximation, only the inhomogenities (IMF) with characteristic dimensions larger than the mean free path length, are efficient for CR variations. The scattering of particles is strong if their gyroradius is smaller than the dimension of the inhomogeneity. The characteristic time of CR variation is the time during which the inhomogeneity, convected with the solar wind speed, is seen at the Earth. For typical quiet conditions the minimum characteristic time of CR variations, according to Bazilevskaya and Struminsky (1993), is 1 hour. That is why in the further analysis, we divided the PSD into two parts, the low frequency one (region $1, f_{1}<f<f_{h}$ ), and the high frequency one (region $2, f_{h}<f<f_{2}$ ), where $f_{1}=1.2$ $\times 10^{-5} \mathrm{~Hz}, f_{h}=2.8 \times 10^{-4} \mathrm{~Hz}$, and $f_{2}=1.7 \times 10^{-3} \mathrm{~Hz}$.

To characterize the PSD, we used the sample spectra, $I^{\prime}\left(f^{\prime}\right)$, rather than periodogram, since $I^{\prime}\left(f^{\prime}\right)$ provides the estimate of the amplitude of sinusoidal component at any particular frequency, $f^{\prime}$, not only at the discrete $f_{i}^{\prime}=i / N$, where $N$ is number of the points in the time series (TS), and $i=1,2, \ldots, N-1$. Let $X_{t}$, $t=1,2, \ldots, N$ is the TS of 5 minute records in one day $(N=288)$. The sample spectra, $I^{\prime}\left(f^{\prime}\right)$, is related to the autocorrelation function $C_{k}$ Box and Jenkins (1970), as

$$
\begin{aligned}
& C_{k}=\frac{1}{N-k} \cdot \sum_{t=1}^{N-k}\left(x_{t}-x\right)\left(x_{t+k}-x\right), \quad x=\frac{1}{N} \cdot \sum_{t=1}^{N} X_{t}, \\
& I^{\prime}\left(f^{\prime}\right)=2 \cdot\left(c_{0}+2 \cdot \sum_{k=1}^{N-1} c_{k} \cdot \cos \left(2 \cdot \pi \cdot f^{\prime} \cdot k\right)\right) .
\end{aligned}
$$

In Eq. (1) $k=0,1,2, \ldots, N-1$. In the basic interval of frequencies, valid for (2), $f^{\prime} \in\{0,0.5\}$. Since the time step in CR measurements is $300 \mathrm{sec}$, we ascribe the sample spectra of CR, $I(f)$, in the real frequencies, $f,\left(f=f^{\prime} / 300\right)$, according to $I^{\prime}\left(f^{\prime}\right) d f^{\prime}=I(f) d f$.

In each of the two frequency regions ( $m=1$ or 2$)$, two kinds of PSD characteristics were computed for each day and each station: (i) $I_{m}(f)$ is fitted by the least squares method, as

$$
I_{m}(f)=c_{m} \cdot f^{-v_{m}},
$$

thus $v_{m}$ as well as the correlation coefficient $r_{m}$ measuring the quality of the fit are obtained; and (ii) integrals

$$
P_{1}=\int_{f_{1}}^{f_{h}} I(f) \cdot d f \quad \text { and } \quad P_{2}=\int_{f_{h}}^{f_{2}} I(f) \cdot d f
$$

are computed. The correlation coefficients $\rho$ of different fluctuation characteristics at the two NMs are given in Table 1 ( 685 days).

In the high frequency region (2) both $P_{2}$ and $v_{2}$ are weakly correlated. The better correlation in $P_{1}$ than in $v_{1}$ is related with more complicated character of PSD than $v$ in a simple power-law approach $\left(f^{v}\right.$ in the whole interval of $f$ ). Additionally, the effect of Poisson noise is contributing to the obtained power spectrum at high frequencies, as it was shown previously by Fujii et al. (1973). For our case, using the relation for the Poisson noise level from Fujii et al. (1973), the level is about $0.075(\%)^{2} /($ cycle per 10 $\mathrm{min})$. This value is still below the lowest values of PSD at high frequencies in Fig. 1, however the bending 
Table 1.

\begin{tabular}{ccccc}
\hline Parameter & $P_{1}$ & $v_{1}$ & $P_{2}$ & $v_{2}$ \\
\hline$\rho$ & 0.89 & 0.38 & 0.14 & 0.05 \\
\hline
\end{tabular}

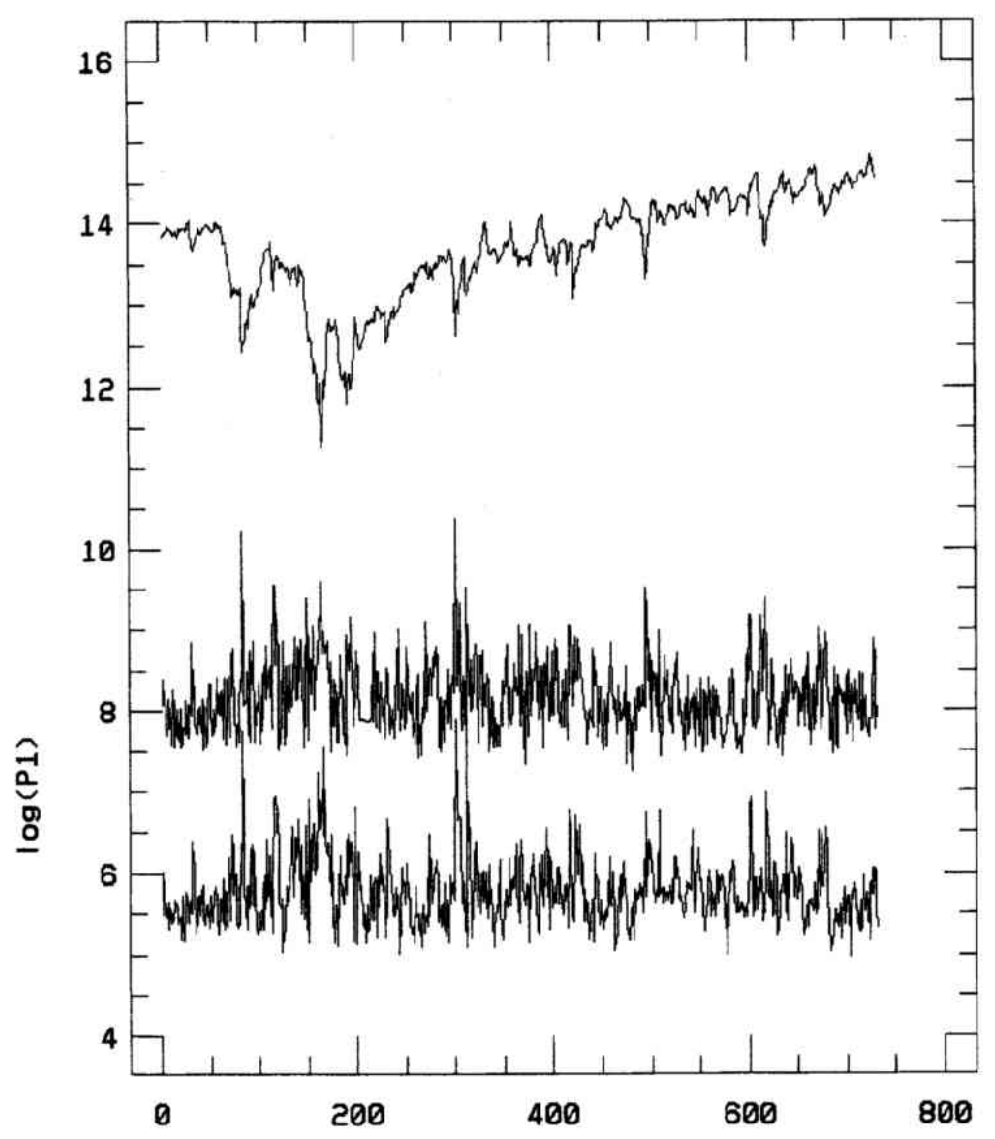

days from 01.01 .1991

Fig. 2. Daily values of $\log \left(P_{1}\right)$ for 1991-1992. The central curve is for LS, the lower one is for CG (shifted by -2 ). For comparison the daily means of CR count rate at CG are displayed (the upper curve), the unity at $y$ axis corresponds to $10 \%$ variation. Days are numbered from January 1, 1991 (day 1).

of the spectra to more flat pattern at $f>2.8 \times 10^{-4} \mathrm{~Hz}$ is probably due to the increasing influence of that effect with increasing frequency. For assuming the high frequency part of the spectra, the technique proposed by Fujii et al. (1973) is suitable to remove the Poisson noise. In the present study, we concentrate ourselves on the frequency region in which the cosmic ray particles are scattered by the interplanetary magnetic field inhomogeneities (frequencies lower than $f_{h}$ ). In that interval the PSD is well above the Poisson noise level. 
Figure 2 shows the time profile of values $P_{1}$ at the two stations along with the count rate of NM Calgary for the period studied. Although the largest values of $P_{1}$, simultaneously observed at CG and LS, are seen often in conjunction with sharp CR decreases, the correlation between $P_{1}$ and CR intensity itself, is low ( 0.16 for CG and 0.14 for LS). Thus, $P_{1}$ can be assumed as a parameter of CR fluctuations commonly characterising PSD at NM energies, which is weakly correlated with CR intensity itself.

\section{Relation of $P_{1}$ to Geomagnetic Activity}

The variations of CR are not affected only by "local" interplanetary state (IMF and solar wind measured near the Earth's orbit), but they reflect the distribution of IMF inhomogenities within the heliosphere. Redistribution of IMF inhomogenities, induced from the Sun, can be thus sensed by CR earlier than e.g., interplanetary travelling shock is reaching the Earth and consequently a geomagnetic disturbance occurs. Earlier, Sakai (1986) has shown that there exists the correlation between the power spectral density of cosmic rays and of Dst.

We checked the cross-correlation between TS of daily values $P_{1}$ and of the geomagnetic indicies $(A p$ and daily averages of $\overline{K p}$ and $\overline{D s t})$. The cross correlations with time lags \pm 5 days are shown in Fig. 3 . The cross correlation functions are asymmetric with respect to lag $=0$. A systematical bias with maximum shifted to positive time lags is seen. This indicates the possibility of stronger relation between averaged geomagnetic indicies on day $i$ to $P_{1}$ obtained from the day $i-1$, than to $P_{1}$ at day $i$. For $\overline{D s t}$ this is seen at both stations and for $\overline{K p}$ and $A p$ it is pronounced little bit more at LS. On the contrary, if CR intensity itself is used instead of $P_{1}$, the cross correlations are either symmetric around lag $0(\overline{K p}$ and $A p)$.

Results of more detailed cross-correlations between $P_{1}$ and Dst are shown in Fig. 4. Here TS of $P_{1}$ is constructed for each hour from the 24-hour interval, and compared with TS of hourly values of $D s t$. It is clearly seen that the $D s t$ hourly values have larger correlation with the values $P_{1}$ deduced from 24 hour interval before (maximum is situated even at time lag $30 \mathrm{hrs}$ ), than with some "current" daily averaged value (time lag $=12 \mathrm{hrs}$ ). The difference in the two correlation coefficients is statistically significant at confidence level 0.95 .

Prediction of $D s t$ based on a set of the solar wind plasma and magnetic field data, measured 8 hours before the predicted value, was successfully demonstrated by Lundstedt and Wintoff (1994), using the scheme of artificial neural network (ANN). The potential possibilities of CR fluctuation characteristics $P_{1}$, reflecting not only the "local" state on interplanetary medium near Earth, could be tested by inserting $P_{1}$ time series into the scheme of ANN for Dst predictions. The advantage of using $P_{1}$ is that there are several NMs producing continuously the data, while solar wind plasma and IMF parameters, at least for some years, are available with many gaps.

The results of $P_{1}$ are influenced also by the strength of the diurnal variation of cosmic ray intensity which is variable from day to day. Nevertheless this effect has no significant influence on the result of the study: the correlation of the integral $P_{1}$ obtained during the time interval $(t-24, t)$ in hours with the $\operatorname{Dst}(t)$ is significantly larger than the correlation between the the "current" value of $P_{1}(t-12, t+12)$ with the $\operatorname{Dst}(t)$. We suggest this is an indication that strong redistribution of interplanetary magnetic field, to which the cosmic rays arriving to the Earth's orbit are sensitive well before the interaction of irregularity (e.g., travelling interplanetary shock) with the geomagnetic cavity, is indicated by the results of the presented study.

\section{Conclusions}

Extensive statistical study ( 2 years of $5 \mathrm{~min}$ CR data at two neutron monitors, including intervals of large interplanetary disturbances) has revealed that:

a) CR fluctuations at NM energies in the frequency range $\left(1.2 \times 10^{-5} \mathrm{~Hz}<f<2.8 \times 10^{-4} \mathrm{~Hz}\right) \mathrm{can}$ be commonly described by $P_{1}$ rather than the power-law index $v_{1} . P_{1}$ is weakly correlated with CR intensity itself. There is only small correlation of PSD at the two stations for $f>2.8 \times 10^{-4} \mathrm{~Hz}$. 

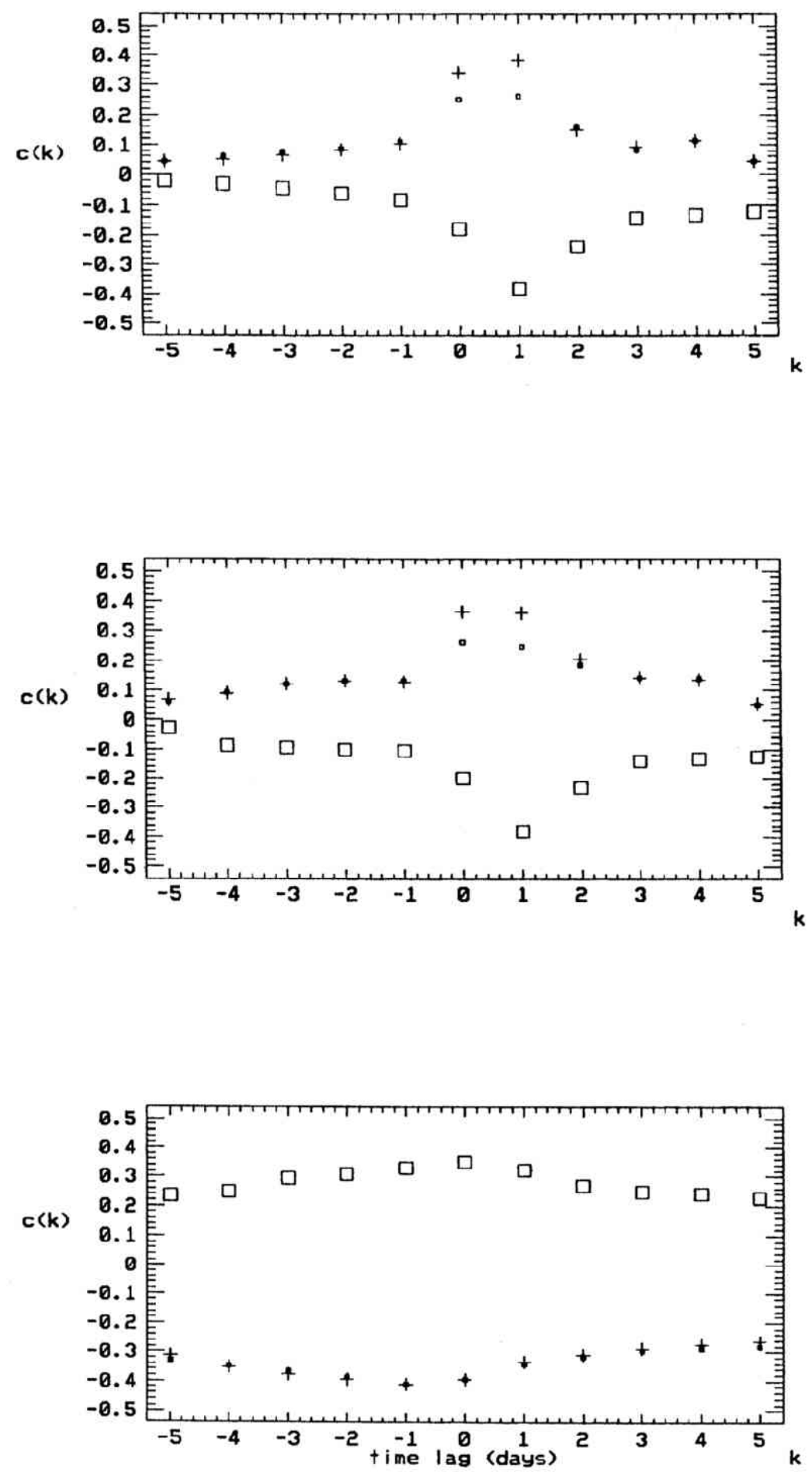

Fig. 3. Cross-correlations, $c(k)$, for the time lags $k \in<-5,+5>$ days, between the time series of daily values of $P_{1}\left(X_{t}\right)$ and the daily averaged geomagnetic indicies $\left(Y_{t+k}\right)$. For the time series $\left(Y_{t+k}\right)$ the values of $\overline{K p}(\bullet), \overline{D s t}(\square)$ and $A p(+)$ for $1991-1992$ are used. The upper panel is for LS NM, the central one for CG NM. In the lower panel, daily means of CR count rate at CG, instead of $P_{1}$, are used as $X_{t}$. The cross-correlation at time lag $=k$ is the correlation of the two time series $\left(X_{t}, Y_{t+k}\right)$. 


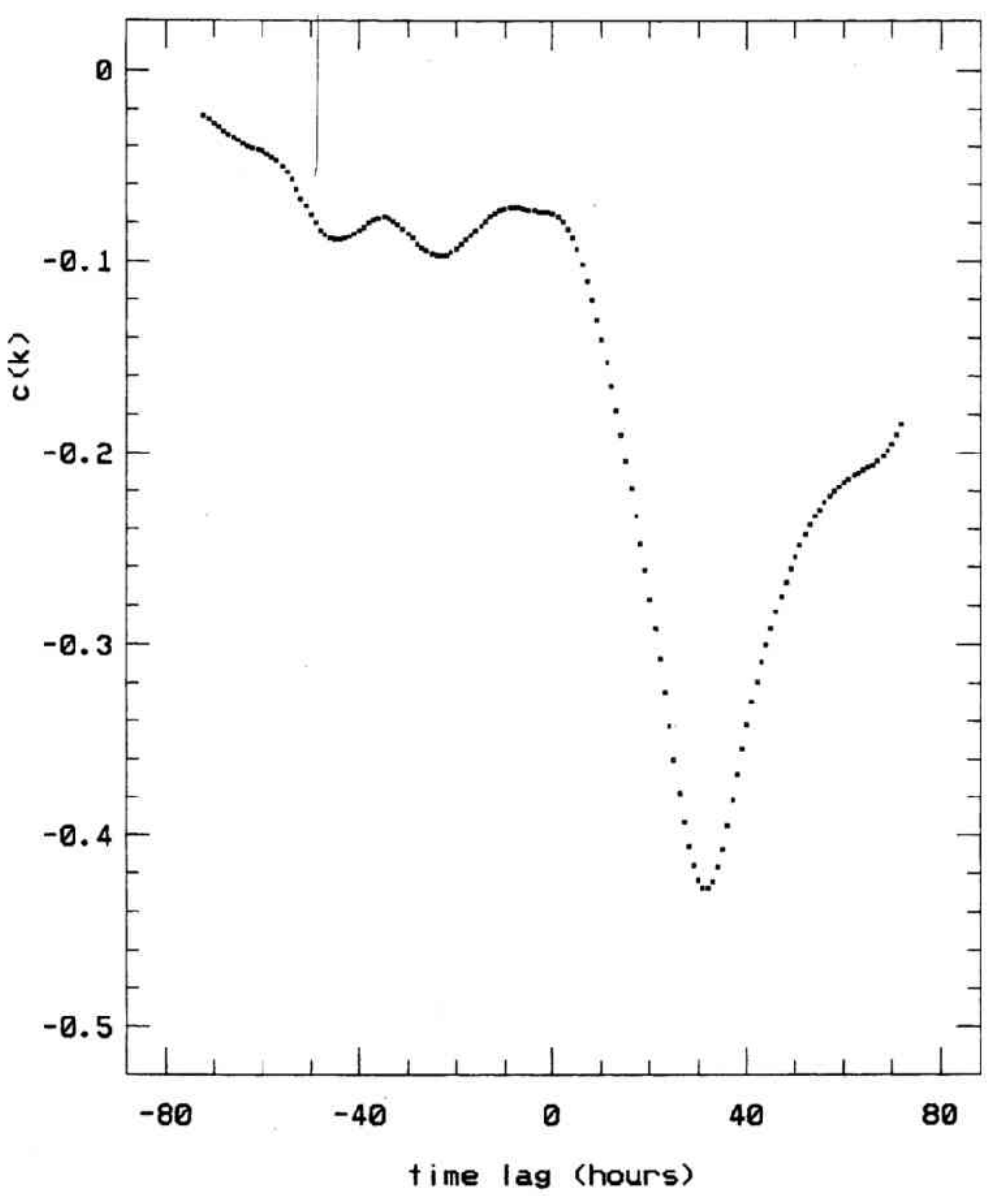

Fig. 4. Cross-correlations, $c(k)$ between the values $P_{1}$, computed from the interval $(t, t+24)$ hours at Lomnicky Stit $\left(X_{t}\right)$ and the Dst hourly values at time $t+k\left(Y_{t+k}\right)$.

b) The cross-correlation of $P_{1}$ with daily averages of $\overline{K p}, \overline{D s t}$ and $A p$, revealed the potential relevance of using $P_{1}$ for the purpose of prediction of geomagnetic activity. We suggest to include $P_{1}$, based on several NM measurements, as one of the parameters, into the scheme of geomagnetic activity predictions using the ANN approach.

The authors wish to thank the referees for their help in evaluating the paper and for important comments.

\section{REFERENCES}

Bazilevskaya, G. A. and A. B. Struminsky, Comment on the characteristic time of cosmic ray intensity variations, Proc. 23rd Int. Cosmic Ray Cont., Calgary, 3, 743-749, 1993.

Box, G. E. P. and G. M. Jenkins, Time Series Analysis, Forecasting and Control, Holden-Day, San Francisco, 1970.

Dhanju, M. S. and V. A. Sarabhai, Short-period variations of cosmic ray intensity, Phys. Rev. Lett., 19, $252-254,1967$.

Fujii, Z., S. Mori, S. Yasue, and K. Nagashima, Power spectrum of the cosmic ray intensity variation, Proc. of the ICRC, Denver, 2, 783-788, 1973.

Jokipii, J. R., Stochastic variations of the cosmic rays in the solar system, Astrophys. J., 156, 1107-1116, 1969.

Jokipii, J. R. and A. J. Owens, Cross-correlation between cosmic ray fluctuations and interplanetary magnetic field fluctuations, Geophys. Res. Lett., 1, 329-332, 1974. 
Lundstedt, H. and P. Wintoff, Prediction of geomagnetic storms from solar wind data with the use of a neural network, Ann. Geophysicae, 12, 19-24, 1994.

Owens, A. J., Cosmic ray scintillations: 2. General theory of interplanetary scintillations, J. Geophys. Res., 79, 895-906, 1974. Owens, A. J. and J. R. Jokipii, Cosmic ray scintillations. 1. Inside the magnetosphere, J. Geophys. Res., 77, 6639-6655, 1972.

Owens, A. J. and J. R. Jokipii, Cosmic ray scintillations: 3. The low-frequency limit and observations of interplanetary scintillations, J. Geophys. Res., 79, 907-912, 1974.

Sakai, T., A relation between power spectral density of cosmic rays and $\overline{D s t}$ field, J. Geomag. Geoelectr., 38, $275-278,1986$.

Shea, M. A., D. F. Smart, D. B. Swinson, and M. D. Wilson, High energy cosmic ray modulation in March-June 1991, Proc. 23rd Int. Cosmic Ray Cont., Calgary, 3, 735-738, 1993. 